\title{
Guerres de sépultures de saints et luttes pour une sacralisation des territoires au Maghreb
}

\section{Kamel Filali}

\section{(2) OpenEdition}

12 Journals

Édition électronique

URL : http://journals.openedition.org/assr/1073

DOI : $10.4000 /$ assr. 1073

ISSN : $1777-5825$

Éditeur

Éditions de l'EHESS

\section{Édition imprimée}

Date de publication : 1 juillet 2003

Pagination : 121-127

ISBN : 2-222-96737-6

ISSN : 0335-5985

\section{Référence électronique}

Kamel Filali, «Guerres de sépultures de saints et luttes pour une sacralisation des territoires au Maghreb ", Archives de sciences sociales des religions [En ligne], 123 | juillet - septembre 2003, mis en ligne le 25 octobre 2005, consulté le 03 mai 2019. URL : http://journals.openedition.org/assr/1073 ; DOI : 10.4000/assr. 1073 


\section{GUERRES DE SÉPULTURES DE SAINTS ET LUTTES POUR UNE SACRALISATION DES TERRITOIRES AU MAGHREB*}

On ne saurait comprendre le phénomène extraordinaire de guerres de sépultures, qui marqua le Maghreb historique, sans passer en revue les différents aspects du culte de la tombe. Très lié à la religion du terroir et étroitement solidaire du système social, il s'ajuste et s'érige comme repère principal autour duquel gravitent de multiples pouvoirs et s'organise la cité maghrébine.

Les tombes des personnages mythiques, symboles de révérence et de puissance, contribuèrent à la naissance et à l'essor des plus vieilles cités méditerranéennes. C'est ainsi qu'elles furent, depuis la nuit des temps, au centre des conflits inter-cités. Hérodote nous apprend que Naxos, selon les oracles, purifia l'île de Crète en déterrant les morts des autres pour ne conserver uniquement ou ne faire vénérer que les siennes parmi les divinités (1).

Le tombeau qui perpétue le pouvoir sanctificateur d'un saint ou d'une idole quelconque est le lieu détenteur d'une gamme de privilèges nombreux. Donnant consistance à certains mythes et symboles, il génère un pôle de pouvoir spirituel et politique et manifeste la force réelle et virtuelle de la tribu. Ses prérogatives relèvent de l'espace cosmogonique et anthropologique (2). Cela dit, en reliant ciel et terre, en jouant le rôle d'intercesseur entre divinité et humanité, il actionne et oriente les mouvements sociodémographiques, comme il regroupe les mythes et les croyances d'inspiration culturelle.

Les privilèges du patronage des tombes ne se limitent pas au côté subjectif et aux formes spiritualistes et cultuelles ; car, le sanctuaire n'implique pas seulement le patronage des esprits, mais aussi beaucoup d'autres légitimités comme celles des territoires immédiats. Agrégats d'habitations, de tribus et de confédérations, chacun dispose de son saint patron. Comme d'ailleurs toutes les villes ont leur patron veilleur ('assâsîn) : ainsi en est-il de Sîdî al-Shaykh patron de al-Bayd, Ibn Shâ'a des

* Note de recherche rédigée à partir d'enquêtes menées dans différentes régions de l'Algérie dans le cadre d'une enquête topographique et anthropologique sur les tombeaux de saints.

(1) Cf. Hérodote, Histoires, tome I, Paris, Éditions Les Belles Lettres, 1970, pp. 64-65.

(2) Mircea Éliade, Le Sacré et le Profane, Paris, Gallimard, 1965, p. 38. 
Beni Zarwâl, dans l'Oranais, Sîdî Bel 'Abbâs, patron de Marrakech, Sîdî al-Huwwârî d'Oran, Sîdî Boumediene de Tlemcen, Sîdî Mahrâs (connu communément sous le nom de sultân al-madîna, le sultan de la ville) est patron de Tunis, Sidi Khâled des Oulâd Djalâl, Yemma Gourâya, protectrice de Bejaïa et sa sœur Lalla Mezghitane, patronne de Jijel, etc (3).

Mythes et légendes rivalisent de merveilleux; ils se répètent, sans cesse, avec comme principale source, le désir de surpasser le voisin en intensité sacrale. Certaines traditions recueillies lors de nos enquêtes illustrent bien les conflictualités intertribales autour des tombes : chaque village tient à célébrer son patron et à en faire le saint le plus distingué et le plus crédible en baraka. Cela donnait souvent lieu à des luttes de préséance.

Comme cela s'est produit dans le Sahara lorsque le corps de Sidi Ali ben Muhammad donna lieu, à la fin du XVI ${ }^{\mathrm{e}}$ siècle, à une bataille entre les tribus de Sîdî Khâled et celle de Oulâd Djallâl. Après des décennies de conflits les Oulâd Khâled l'emportèrent sur ces derniers et gardèrent le tombeau de Sîdî Khâled sur leur territoire, ce qui obligea les Ouled Djalâl à se faire dépendants, faute de repères sacrés, de la tombe du saint patron de leurs adversaires. (4)

La signification d'une tombe ou des reliques d'un saint renvoit, semble-t-il, plus à une stratégie de pouvoir qu'à une pratique superstitieuse. Elle accentue le droit de la propriété de la tribu sur des territoires précis et la consécration des espaces qui l'entourent. C'est pour cela d'ailleurs que les tombeaux de saints particulièrement influents devenaient des enjeux de rivalités entre tribus. Plus le marabout appartenait à une des lignées chérifiennes les plus renommées (Idrissides, Quraychites ou Fatimides) ou était affilié à la grande chaîne initiatique des maîtres soufis les plus éminents en dons et en connaissance spirituelle (al-haqîqa), plus il était au centre de conflits et de rivalités entre clans. Sîdî Boumediene Shu'ayb (né à Séville en 1126, mort à Tlemcen en 1262), a voulu de son vivant détrôner al-Ubbâd (les adorateurs) en prédisant qu'il mourait et serait enterré sur la même terre qu'eux. Vœu qui s'est effectivement réalisé puisqu'il est devenu par la suite un concurrent posthume. Bien que le lieu ait gardé le nom des dits saints, le patronage de Tlemcen fut détourné à jamais au profit de Sîdî Boumediene Shu'ayb.

Pierre angulaire de l'existence de la tribu, le sanctuaire allait contribuer amplement aux changements sociaux et aux mouvements démographiques. Il devint le lieu de fixation, puis de sédentarisation d'un groupe nomade ou d'une tribu vivant de transhumance. Dès lors, il se produit un brassage entre les différents segments de tribus, jusqu'alors étrangers les uns aux autres. Ainsi, les gens de Touggourt se sont sédentarisés autour du tombeau de leur patronne, Lalla al-Bahdja (Notre Dame la joyeuse) une convertie, vers la fin du $\mathrm{XV}^{\mathrm{e}}$ siècle, par Sîdî Yahyia, un saint marocain revenant de la Mecque. Une des plus grandes fractions de la tribu nomade des Oulâd Naïl, les Ouled Harkât, qui s'adonnait au commerce se fixa finalement autour du tombeau de Sîdî Khâled situé à quatre-vingts kilomètres environ au sud-est de Biskra, aux abords de Wâd Jdî.

(3) Sur le personnage cf. Émile Dermenghem, Le Culte des Saints dans l'islam maghrébin, Paris, Gallimard, 1954, pp. 162-164.

(4) Entretien effectué, en mars 1992, à Sîdî Khâled. 
Comme on le voit, le sacré constitue le plus souvent un facteur de paix et de cohésion. Mais il peut aussi être la source de mésententes, de rivalités et de manipulations à des fins politiques. Ainsi, en est-il de la Kabylie, lorsque les saints populaires arrivent à se constituer en autant de chapelles rivales sur des territoires et des enclaves bien délimités connus sous le nom d'Imrâbtan. Ces territoires sont fréquemment déployés dans la plaine voisine du massif des Montagnes du Djurdjura $(2308 \mathrm{~m})$. De cette rivalité, il en est résulté des sobriquets qui distinguent les marabouts d'Oufalla (ceux du haut) et les marabouts d'en bas, se nommant Aoudda. Les marabouts se prévalant d'un caractère noble, s'efforcent de disqualifier les aguellids animistes pour mieux les détrôner de leurs positions sociales et de leurs influences traditionnelles dans les tribus de la Berbérie antique.

Le marabout souvent prosélyte issu d'une des trois pépinières de la noblesse religieuse, à savoir : al-Saqiyya al-Hamra (Sahara occidental) Oued Dra', Tafilalt, et Fes (Maroc), en quête de terroir, se voit reléguer dans le rôle du fondateur des Aoudda, en raison du mépris que lui vouent les Oufalla prétendument nobles. Il est arrivé parfois que la tribu berbère des Ouffalla, assoiffée de sacralisation se fasse «maraboutisée » en acceptant l'établissement de l'anachorète sur ses territoires. C'est le cas du village de Sadouk Ouffalla qui porte le nom de l'ancêtre éponyme de la lignée des fondateurs mythiques et insurgés, les fameux Haddâd, chefs de fils de la résistance anticoloniale en Kabylie (1871-1881).

La sépulture constitue le point focal et le centre vers lequel convergent non seulement les regards, mais aussi tous les consommateurs du culte des saints. Elle fonctionne également comme un facteur de propagation symbolique et de rassemblement des éléments épars telles des tribus naguères éloignées par le lignage ou rivales. Mais avec le temps, la sépulture finit par effacer les différences entre les différents membres de la tribu ou la fraction de tribu; elle cimente et resserre les liens de nature affective, spirituelle et religieuse. Elle abolit la différence liée à l'origine ethnique, sociale et d'appartenance spécifique. La sépulture va de pair également avec le culte de l'ancêtre car celui-ci est d'autant plus sanctifié qu'il est associé dans l'imaginaire social à un véritable saint, et comme tel, il est perpétué par des ziyâra et des offrandes périodiques.

Sîdî Ahmad al-Zawâwî qui naquit vers la fin du XVIII siècle à Mila (à l'ouest de Constantine), est un exemple typique de ces ancêtres vénérés et sanctifiés.

Pressentant sa mort prochaine, il fit le vœu de se faire ensevelir sous un palmier chez les Ouled Bouhamma. Ses adeptes exaucèrent ses vœux. Mais non contents de ce geste et, comme par jalousie, ils désirent s'accaparer à leur seul profit la dépouille, les Ouled Ben al-'Attâr (5), une des plus vieilles noblesses de la région, s'empressent de le déterrer nuitamment pour l'enterrer à Mila al-qadîma. Contre toute attente, cette dernière ne conteste pas l'acte frauduleux, mieux encore elle accepte de fusionner avec la sainte tribu adverse (6).

Le tombeau devient ainsi sujet et objet de cultes et de pouvoirs magiques aux mains de multiples monarques. Le sanctuaire sert de liens de dépendance et d'inter-

(5) Cette appellation qui signifie vendeurs de parfum est portée également par une lignée de saints juifs marocains ; cf. Issachar Ben AMI, Culte des Saints et pèlerinages judéo-musulmans au Maroc, Paris, Maisonnneuve et Larose, 1990.

(6) Tradition recueillie auprès du cheikh Hocine Ben al-'Attâr. 
dépendance entre le spirituel et le profane et définit en même temps les rapports réciproques des différents groupes en compétition.

Ces rapports peuvent prendre une forme conflictuelle, susciter des tensions, mais elle n'exclut pas des concessions et des échanges d'intérêts entre vénérant et vénéré. Interrogeant un pèlerin de Sîdî Mûl al-Kâf, Monseigneur le patron du Rocher, une divinité anonyme et immémoriale au douar Taïlmam (El-Ancer, dans les environs de Jijel), sur les visites pieuses et l'aumône, celui-ci répondit: " al-çadâqa fì al-wâlî wa la fî al ma'rûf (L'aumône sera mieux affectée au saint qu'à un familier nécessiteux) » (7). Le cumul d'offrandes convertit ces tombes en lieux de commerce et de souks hebdomadaires où l'on vient troquer des biens et invoquer la baraka pour obtenir des dons divins (rizq). La Tombe de Sîdî Daoud (Dellys) sur la côte kabyle était, pendant toute l'époque ottomane (1519-1830), un centre de négoce de sel où chaque négociant, peu importe sa confession, juive, chrétienne ou musulmane, doit offrir (haqq al-ard), une quantité de sel en guise d'offrande.

Les luttes entre saints pour des places stratégiques sont très suggestives et laissent entrevoir des arrières pensées de pouvoir. Tout le long de leurs tracés historiques, les entités maghrébines étaient vouées à un mythe fondateur (8), à une providence salutaire ou à une sacralité sécurisante et fertilisante. Elles se font et se défont selon les rapports de force entre telle ou telle divinité.

La terre sur laquelle repose le saint de la tribu est déclarée et reconnue harâm, défendue à ceux qui ne croient pas en son pouvoir et à ses ennemis. Elle est souvent délimitée par des kerkours, sortes de jalons en pierres. Le hrm (droit sacré) accordé aux restes de la dépouille exprime la grande vénération et le zèle de l'idole (9).

Les conflits autour du choix du lieu de l'enterrement d'une sainte sépulture cachent souvent une arrière-pensée, en l'occurrence commerciale, voire mercantile. Selon la légende, Sîdî 'Ammâr, marabout établit aux alentours de Ghardaïa, fut chargé par les Oulâd Saïd, une tribu bédouine de rite malékite, de régler leurs différends résultant d'un butin de guerre au détriment des mozabites (de rite kharijite). Il fit un partage équitable entre les deux partis brouillés ; et, de cette action salutaire, il s'acquit une légitimité de saint patron sur une confédération tribale. Avant de mourir, nous dit la légende, il adopta un enfant qu'il trouva abandonné auprès d'un laurier rose (al-dafla), nom qu'il attribua à l'enfant qui devint son éponyme post-mortem, Sîdî mûl al-Dafla. Les tribus dont il est issu fréquentaient alors le marché de Touggourt et contribuèrent à la prospérité du commerce de cette ville. À sa mort, elles créèrent leur propre marché, qui sera d'autant plus attractif qu'il sera plus sanctifié. Inquiets de l'essor de leur commerce, les sages Touggourtins recoururent à cette ruse de bilocalisation miraculeuse de la sépulture de Sîdî 'Amar (10).

La mort d'un saint, lors d'une siyyâha (un voyage d'initiation et de quête de connaissance), loin de sa tribu est considérée comme une fatalité. Désanctificatrice,

(7) Tradition recueillie auprès de la dite tribu.

(8) Cf. Kamel Filali, Quelques éléments du mythe de l'origine de l'Algérie ottomane, in Mélange Charles Robert Ageron, Tunis, Fetersi, 1996.

(9) Sur le Hrm voir J. Chelhod, Les Structures du sacré chez les arabes, Paris, Maisonneuve et Larose, 1969.

(10) Tradition recueillie auprès du chérifien al- hadj al-'Ulmî, tribu ouled said (Touggourt). 
elle peut engendrer la perte de toute considération et par conséquent de tout intérêt économique ou profane. Elle pourra aussi causer à ses atbâ' des désordres dans le culte qui, quelquefois, provoquaient la ruine de la tribu. Sîdî Ahmad ben 'Amâr, fils de l'illustre Ahmad al-Tidjânî (le fondateur de la Tijâniya) décéda en 1837, non loin de la frontière tunisienne, près de Gmâr. Les gens de cette localité se hâtèrent de l'enterrer avant même que l'écho de sa mort ne parvînt à Aïn Madhi. Au su de cet événement, ses adeptes inquiets accoururent de nuit, le déterrèrent et le prirent à Ain Madhi. Évitant un conflit avec leurs congénères, les habitants de Gmâr qui étaient en majorité affiliés à la Tidjâniya, se contentant des odeurs de sainteté, observaient un dédoublement miraculeux de la tombe. Parfois quand une tribu se fait dépouiller de sa sainte sépulture et qu'elle n'est pas en mesure de rivaliser, elle se résigne au fait extraordinaire de dédoublement de la tombe.

La sacralisation par les éléments fétiches de la tombe peut servir de moyen potentiel d'anoblissement religieux, en l'occurrence chérifien. Cette modalité est largement utilisée par les tribus hillaliennes « laïques » qui pourtant optèrent pour une "maraboutisation » généralisée. Quand elles sont privées de baraka, les tribus bédouines d'origine hillalienne (11) sont vulnérables et se retrouvent plus exposées aux appétits des voisins. C'est alors qu'elles se dérobent et se cachent derrière une sacralisation inflexible en trafiquant des reliques ou en permettant l'établissement d'un marabout chérifien (afférent à la lignée généalogique du Prophète de l'islam). Sîdî Mokhtar, arrière-grand-père de l'Émir Abd al-Kader (1808-1883) mort chez la tribu bédouine des Béni 'Âmer (dans l'Oranais), en avait fait ainsi. Soucieux de l'enterrer près de leur zawiya de Casharou, dans le cimetière familial éponyme de leur saint fondateur, Sîdî Abd al-Kader dit Qada, (décédé à la fin du XVIII ${ }^{\mathrm{e}}$ siècle), son fils, Sîdî Muhammad, emporta le cadavre de nuit en catimini. Mais les Beni 'Âmer tribu turbulente réputée pour le brigandage et les exactions, - toujours en recherche d'une relique pour une sacralisation - proclama un miracle, et prétendit avoir retrouvé son corps. Sîdî Mokhtar devint Bûqabrîn (le saint aux deux tombeaux) et la tribu jouit depuis du statut chérifien (12).

Toutes les opérations, contestées, de déterrement se font de nuit, ce qui révèle leur caractère frauduleux et les arrières-pensés stratégiques qui se trouvent derrière. Ainsi Sîdî Ali ben Aïssa, saint marabout de Férouma (13) un des disciples de Sîdî Muhammad ben 'Abd-al-Rahmân, le fameux Bûqabrîn, enterré à Hamma d'Alger (Belcourt) et à Goudjala, Djurdjura. Les gens de Ferouma ne pouvant se passer de sa sainte et bénéfique sépulture firent rapatrier son corps de nuit pour l'inhumer sur les hautes cimes du Djurdjura dans sa tribu d'origine, non loin des Flissa. Depuis, il est reconnu comme le saint gardien de la localité veillant jour et nuit sur les terres et les cieux qui entourent les territoires de la tribu. Cet enlèvement ne causa aucune perte d'influence ni de rayonnement. Fort heureusement, l'ancienne tombe déjà radiée jouissait toujours de prérogatives et de vénérabilité (14).

(11) Elles campaient jadis à l'est du Nil et furent envoyées, en 1055, pour punir les dissidents Fatimides du Maghreb.

(12) Émile Der Menghem, «Les souvenirs de l’Émir Abdelkader dans la région de Mascara », Documentation algérienne, série culturelle, 15/10/1949, p. 118.

(13) Bourgade située aux environs de Lakhdariya à $50 \mathrm{~km}$ d'Alger vers l'Est.

(14) Hamdân Khûja, al Mirât, (chroniques de la ville d'Alger [datant de 1837]) $3^{\mathrm{e}}$ éd., Alger, S.N.E.D, 1982, p. 58. 
Bien que tout le monde rêve de voir mourir un sâyih (pérégrinant) sur son territoire, au Maghreb on ne connaît pas de meurtre commis dans un but d'accaparement de la dépouille d'un saint comme cela se passait au Pendjab (l'Inde) où on invitait le saint voisin pour le tuer et l'enterrer de manière à être sûr qu'il resterait et protègerait le pays (15).

Néanmoins, "la guerre de sépultures » conduit souvent à la discorde incendiaire; elle peut être à l'origine de la dislocation d'un groupe social ou d'une confédération tribale. Non loin de Ferdjiwa, à une cinquantaine de kilomètres à l'ouest de Constantine, au début du XVI ${ }^{\mathrm{e}}$ siècle, Sîdî al-Shaykh al-'Ayâd régnait sur un groupe confédéré de tribus partagées entre les deux rives par l'Oued el-Kébir. Son nom devint éponyme des Ouled Bouffaha après avoir réussi merveilleusement à les souder et à les réunir. À sa mort, il fut enterré sur le côté haut, ce qui provoqua la mésentente de la basse tribu. Les habitants de cette dernière, redoutant une désanctification, décidèrent alors de le déterrer de nuit pour le réenterrer sur leurs territoires. Cet acte frauduleux déclencha une guerre entre les deux factions tribales qui dura, dit-on, dix ans (16).

La guerre entre les Ouled Bouffaha et les Hadriya (tribus situées sur la rive droite de l'oued el-Kébir) à propos de la sépulture de Sîdî Boumerdis dura deux siècles (du XVIII à la colonisation française). Après une longue suite d'altercations, les Ouled Bouffaha arrivèrent à maintenir le sarcophage de leur saint sur leur territoire ; poussant plus loin leur entêtement, ils interdirent même aux Hadriya de parler du songe collectif qu'ils prétendirent avoir fait.

Toutes les congrégations n'ont pas été impliquées dans cette " guerre de sépultures ». Celles dont le pouvoir était le plus limité et qui souffraient des exactions, notamment des tribus maraboutiques voisines, se sont levées avec hostilité pour réfuter toute pratique de culte de la tombe. Pratique qu'ils considéraient comme une innovation blâmable. La confrérie 'Alawiyya, dérivée de la Derqawiyya, qui se trouve encerclée de part et d'autre de Mostaganem par les entités maraboutiques, s'est élevée avec véhémence contre la dévotion faite aux tombeaux qu'elle regardait comme une : « coutume stupide et anti-musulmane » (17). Cette propagation stratégique de dédoublement de sépultures de saints, si contagieuse, était, peut-être, la raison qui avait aussi poussé les mozabites à condamner les saints et le culte des tombeaux en s'enfermant dans leur oasis et en barrant la route à cette forme de spiritualité conquérante.

Kamel FILALI

Université de Constantine p. 125 .

(15) Cf. Westermark, Ritual and Belief in Marocco, I, Cambridge, Cambridge University Press,

(16) Tradition recueillie auprès de la tribu des Marmoul (Ferjiwa).

(17) Sur la 'Alawiya voir: Johan ARTignY, Cheikh Ahmad al-'Alwî, Documents et témoignages, Paris, 1984, p. 35. 
La guerre autour de la sainte sépulture reflète autant le culte d'un personnage vénéré qu'une lutte dont l'enjeu est le prestige et le pouvoir. Chaque tribu tente par l'intermédiaire de son cheikh de s'imposer et de s'étendre au détriment de ses rivaux en faisant valoir la noblesse ou le caractère prééminent et sacré de son saint favori. La sépulture qui l'abrite est réputée plus vénérable ou plus prestigieuse que celles des tribus ou villages voisins. Le tombeau du saint censé abrité la baraka, garante de puissance et de richesse, devient dès lors l'objet d'une rivalité acerbe entre chefs de tribus. Des filiations nobles, réelles ou fictives, sont mises en avant par chacune des tribus prétendant à l'hégémonie ou à la prééminence sur les autres.

\section{Abstract}

The fight over holy sepultures reflects both the cult of a revered figure and a struggle for prestige and power. Through the intermediary of its sheik each tribe tries to impose itself and extend its territory at the expense of its rivals by claiming the nobleness and the pre-eminent and sacred character of its favorite saint. His burial place is said to be more venerable or more prestigious than those of the neighbouring tribes or villages. The saint's tomb which is supposed to shelter the "baraka" , source of power and wealth, becomes the object of an intense rivalry between the tribes' chiefs. Real or fictitious noble origins are claimed by each of the tribes who intend to impose its hegemony or pre-eminence over the others.

\section{Resumen}

La guerra alrededor del santo sepulcro refleja tanto el culto de un personaje venerado como una lucha cuya apuesta es el prestigio y el poder. Cada tribu trata, por intermedio de su sheik, imponerse y extenderse a costas de sus rivales, haciendo valer la nobleza o el carácter preeminente y sagrado de su favorito. La sepultura que lo abriga es considerada más venerable o más prestigiosa que las de las tribus o los pueblos vecinos. La tumba del santo, que contiene supuestamente la baraka, garantía de poder y de riqueza, se convierte entonces en el objeto de una rivalidad enconada entre jefes de tribus. Filiaciones nobles, reales o ficticias, son destacadas por cada una de las tribus, que pretenden la hegemonía o la preeminencia sobre las otras. 